Ming Li

Theory of Fractional Engineering Vibrations 


\section{Fractional Calculus in Applied Sciences and Engineering}

Editor-in Chief

Changpin Li

Editorial Board

Virginia Kiryakova

Francesco Mainardi

Dragan Spasic

Bruce Ian Henry

YangQuan Chen

\section{Volume 9}


Ming Li

Theory of Fractional Engineering Vibrations

DE GRUYTER 
Mathematics Subject Classification 2010

Primary: 34-xx, 34Axx, 34Mxx, 45Gxx, 65Lxx, 70Jxx, 70Kxx, 74Dxx; Secondary: 26A33, 34A37, 34C10, 34C15, 34C26, 34K11, 70P05, 74H45, 74H55

\section{Author}

Dr. Ming Li

Zhejiang University

Ocean College

Ocean Engineering Building

316021 Zhoushan

PR. China

East China Normal University

200241 Shanghai

PR. China

ming_lihk@yahoo.com

ISBN 978-3-11-072652-7

e-ISBN (PDF) 978-3-11-072615-2

e-ISBN (EPUB) 978-3-11-072616-9

ISSN 2509-7210

Library of Congress Control Number: 2020951347

Bibliographic information published by the Deutsche Nationalbibliothek The Deutsche Nationalbibliothek lists this publication in the Deutsche Nationalbibliografie; detailed bibliographic data are available on the Internet at http://dnb.dnb.de.

(C) 2021 Walter de Gruyter GmbH, Berlin/Boston

Cover image: naddi/iStock/thinkstock

Typesetting: VTeX UAB, Lithuania

Printing and binding: $\mathrm{CPI}$ books $\mathrm{GmbH}$, Leck

www.degruyter.com 
To Yonglang Zhang and Joanna Jiayue $\mathrm{Li}$ - for making it both possible and worthwhile 
